\title{
An aminotransferase from Enhydrobacter aerosaccus to obtain optically pure $\beta$-phenylalanine
}

Xinming Feng ${ }^{\dagger},{ }^{\ddagger}$, Jing Guo ${ }^{\dagger}$, Rubing Zhang ${ }^{\dagger}$, Wei Liu ${ }^{\dagger}$, Yujin Cao* †and Mo Xian* †

${ }^{\dagger}$ CAS Key Laboratory of Biobased Materials, Qingdao Institute of Bioenergy and Bioprocess Technology, Chinese Academy of Sciences, Qingdao, China

tUniversity of Chinese Academy of Sciences, Beijing, China

Correspondence:

Yujin Cao, Email: caoyj@qibebt.ac.cn

Mo Xian, Email: xianmo1@qibebt.ac.cn 
Nucleotide sequence of $\omega$-TAEn (codon-optimized)

ATGAGTTGCAGTAGTTGTGGTGCCACCAGTAAAAAAGTGCCGAGTAGCGC AAGTAGCGTTTGGGATAGTTATGAACCGATTAGTGCCGGTCATCTGGTGC GTAAACAGACCGCCTGTGATCGCGTTGCACCGGCCGATGAAGAATGCCGC GGTCGTGTTACCCTGCCGGATCAGGTTCTGGCCAATAGCGATCTGCAGAG CGCACTGGTTGAAGCCCGTCAGGCCTATATTGATCGCAATCCGAAAAGTT TTGCACGTCATCAGGAAGCCAGCCAGGCCATGCCGGGCGGTAATACCCGT ACCACCCTGCATAATGCCCCGTTTCCGCTGACCGTGGTTCGCGGCGAAGG CTGCCGCCTGTGGGATGCAGATGGCCATGAATATATTGATGTGCTGGGCG AATATACCGCCGGTATCTATGGTCATAGCCATCCGGTTATTCGTGCCGCCA TTGATCGTGCACTGGATCATGGCTGGAATTATGCCGGTCGCAATGCATAT GAAGGTGAACTGGCACGCCTGATTGTTGATCGTATTCCGAGCATTGATCT GGTGCGTTTTACCAATAGTGGTACCGAAGGCAATGTGATGGCCCTGGCAG GTGCCAAAGTGTTTGCACAGCGTGCCGGCCGCGCCAAAGCAACCAAAGTG ATGGTGTTTCATGGTGGTTATCATGGTGGTGTGCTGTATTTTGTTAGTGGT GGTAGTCCGGTTAATATGCCGTATGATTATGTGGTTGCCCCGTATAATGAT ATTGAAGGTACCCGTGCACTGCTGGCCAAACATGGTGAAGAAATTTTTGC AGTGCTGGTTGAACCGATGCAGGGCAGTCATGGCTGCCTGCCGGGCGATC CGGATTTTCTGCGTGCACTGCGCGATGAAACCCGTGCACGCGGTATTACC CTGATTTTTGATGAAGTGATGACCAGTCGCCTGAGCCCGGGCGGTCTGCA GGCTAGACTGGGCATTGTGCCGGATATGACCACCCTGGGTAAATATATTG GTGGTGGTATGAGTTTTGGTGCCTTTGGCGGTCGTCGCGAAATTATGGAAC TGTTTGATCCGACCAAACCGGATAGCCTGCCGCATGCAGGTACCTTTAAT AATAATGTGCTGACCATGGCAGCCGGTGTGGCAGGTTATGGCGAAGTTTA TACCCCGGATGTGGCAGATGCACTGAATGCCCGTGGTGACCGTATTCGTG ATCGTCTGAATGCAATTTGTGCAAAAAATGATGTGGCATTTCAGTTTAGCG GTATTGGTAGCATGATGACCGCCCATGCAACCACCCGTCCGATTCGCACC GCCGCAGATATTGCCAGTAGCAATCAGGATGCCAAAGAACTGTTTTTCTTT GATATGAGCGCAGCAGGCATTTGGATTGCCCGTCGCGGCTTTGCCGCCCT GAATATTATGATTGGCGAAGCCGAAGCAGATCGTTTTGTTAGTGCCGTTG AAGAATTTGTTACCGCACGTAAAGCACTGCTGCAGCCG

\section{$\beta$-Phenylpyruvic acid preparation}

$80 \mathrm{mM}$ ethyl phenylpyruvate was hydrolyzed with $80 \mathrm{~mL} \mathrm{NaOH}(1 \mathrm{M})$ in a $250 \mathrm{~mL}$ Erlenmeyer flask. Then $10 \mathrm{~mL}$ methylene dichloride was used to remove the residual esters for three times. The aqueous phase was cooled by ice bath to reduce by-products. $3 \mathrm{M} \mathrm{HCl}$ was used to adjust $\mathrm{pH}$ to 1 and then $\beta$-phenylpyruvic acid, a pale yellow solid, was obtained. 
Figure S1 Amino acid alignment among the $\omega$-TAEn and other related protein generated by CLUSTAL O(1.2.4)

\begin{tabular}{|c|c|c|c|}
\hline TAMe & 1 & 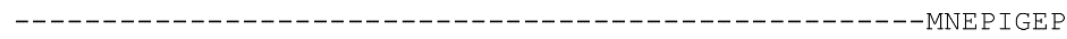 & 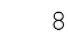 \\
\hline TAPO & 1 & ----------------------------------------------------MNKPS$--\mathrm{T}$ & \\
\hline TAVa & 1 & 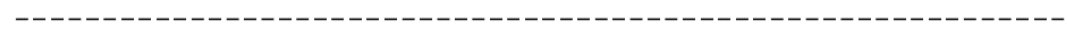 & \\
\hline TAPa & 1 & 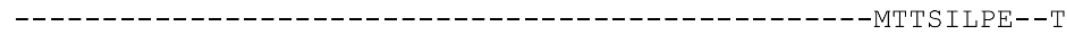 & \\
\hline חת תח & 1 & MSCSSCGATSKKVPSSASSVWDSYEPISAGHLVRKQTACDRVAPADEECRGRVTLPD--- & \\
\hline 2115 & 9 & GRSPASDTAEKAQAIAAARNTFARDNPVSAGHHERARRSMPGGNTRSILFHRPFPLVIAQ & \\
\hline APO & 7 & SSMHSATGTALDSALAETTARYRARNPGSERLLRKAADVLPAGNTRSVLFYTPFPLYMAR & \\
\hline & 1 & -----MTHAAIDQALADAYRRFTDANPASQRQFEAQARYMPGANSRSVLFYAPEPLTIAR & \\
\hline & 10 & QVHARSDAATVGEALAAAAAAFAARNPQSÃQHYERÃTEVMPGGNTRSVLFYEPFPLAMAK & \\
\hline \multirow[t]{2}{*}{ TAEn } & 58 & $---Q V L A N S D L Q S A L V E A R Q A Y I D R N P K S F A R H Q E A S Q A M P G G N T R T T L H N A P F P L T V V R$ & \\
\hline & & 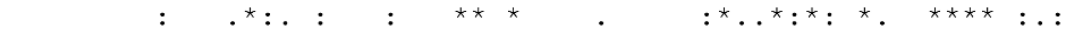 & \\
\hline & 69 & GTGSRFQDVDGHAYVNFLGEYTAGLFGHSHPVIRAAVERALAVGLNLSTQTENEALFAEA & 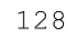 \\
\hline APO & 67 & GEGCHLWDADGHRYLDALGEFTAGIYGHSNPVIRQA IVAALQDGLSLSSHTAREAALAHE & \\
\hline & 56 & GEGAALWDADGHRYADF IAEYTAGVYGHSAPEIRDAVIEAMQ̊GGINLTGHNLLEGRLARL & \\
\hline & 70 & GEGCRLWDADGHEYLDFIAEFSAGIYGHSHPQIRAAIDAALDD GLNLSGHNLLESRLAKA & \\
\hline \multirow[t]{2}{*}{ TAEn } & 115 & GEGCRLWDADGHEYIDVLGEYTAGIYGHSHPVIRAA I DRALDHGWNYA GRNAYEGELARL & \\
\hline & & 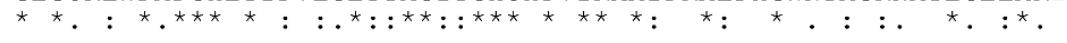 & \\
\hline & 29 & VCDRFPSIDLVRFTNSGTEANLMALATATAITGR------KTVLAFDGGYHGGLLNFASG & 2 \\
\hline & 7 & IQRRFPGMALLRFTNSGTEANLMALAAATAHTGR------RKVLVFNGAYHGGVLSFGGG & \\
\hline & 116 & ICERFPQIEQLRFTNSGTEANLMALTAALHFTGR------RKIVVFSGGYHGGVLGFGAR & \\
\hline & 130 & VCERFASLETVRFTNSGTEANLMMLATAKAFTRR------SKVIVFVGGYHGGVLTFGRG & \\
\hline \multirow[t]{2}{*}{ TAEn } & 175 & IVDRIPS IDLVRFTNSGTEGNVMALAGAKVFAQRAGRAKATKVMVFHGGYHGGVLYFVSG & \\
\hline & & 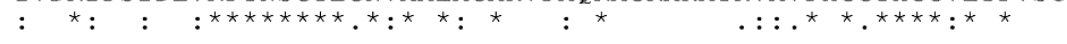 & \\
\hline & 83 & HAPTNAPYHVVLGVYNDVEGTADLLKRHGHDCAAI LVEPMLGAGGCVPAERAFLDLLRAE & 2 \\
\hline & 31 & GSPVNVPHDFVVAPYNDLDAVRGLVQTHGPQLAA I LVEPMLGAGGC I PAEPAFLHGLRAL & \\
\hline & 170 & PSPTTVPFDFLVLPYNDAQTARAQIERHGPEIAAVLVEPMQGASGCI PGQPDFLQALRES & \\
\hline TAPa & 184 & ANVVNVPHPFLYAEYNDLDSVRRLLDENANEVAA I LVEPMQGASGC IVGEPAFLQGLREL & \\
\hline \multirow[t]{2}{*}{ TAEn } & 235 & GSPVNMPYDYVVAPYNDIEGTRALLAKHGEE I FAVLVEPMQGSHGCLPGDPDFLRALRDE & \\
\hline & & 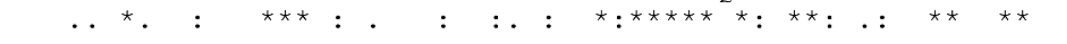 & \\
\hline & 43 & ASRCGALLIFDEVMTSRLSGGGAQEMLGISADLTTLGKYIGGGMSFGAFGGRRDLMERFD & 3 \\
\hline & 11 & ADACGALLILDEVMTSRLSGGGRQALLGLKPDLTTLGKYFGGGLSFGAFGGRVDVMSRFD & \\
\hline & 230 & ATKVGALLVFDEVMTSRLAPHGLANKLGIRSDLTTLGKYIGGGMSFGAFGGRADVMALFD & \\
\hline TAPa & 4 & SSAHGALLMFDEVMTSRLAPGGLQAELGITPDLTSLGKYIGGGMSFGAFGGRAEIMALFD & \\
\hline \multirow[t]{2}{*}{ TAEn } & & TRARGITLIFDEVMTSRLSPGGLQARLGIVPDMTTLGKYIGGGMSFGAFGGRRE IMELFD & \\
\hline & & 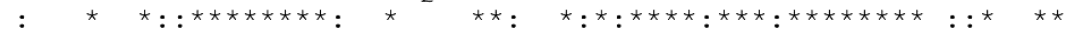 & \\
\hline & 3 & PARDGAFAHAGTENNNILTMSAGHAALTQIYTRQAASDLSASGDRFRANLNRIAVENQAP & 36 \\
\hline & 1 & PRRADALGHAGTFNNNTLTMAAGLAGLTQVLTPAALDALNQRGERLRERLNGVFKRHAVG & \\
\hline & 290 & PR-TGPLAHSGTFNNNVMTMAAGYAGLTKLFTPEAAGALAERGDALRARLNAQCANEGVA & \\
\hline TAPa & & PRRSGALQHAGTFNNNVMTMAAGWTGLTQIFTPEAARALTARGDALREALNSRFAASNVA & \\
\hline \multirow[t]{2}{*}{ TAEn } & & PTKPDSLPHAGTFNNNVLTMAAGVAGYGEVYTPDVADALNARGDRIRDRLNA ICAKNDVA & \\
\hline & & 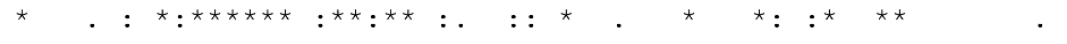 & \\
\hline & & LQFTGLGSLGTIHFSRAPIRSAGDVRAADQQLKELFFFHMLRKGIYLAPRGMYALSLEIA & \\
\hline & & LQFTGLGSVMQLHATDRPLRNAADLAGADDRVKALLFFDLLERGIFLARRGLVALSLPFG & \\
\hline & & MQFTGIGSLMNAHFVQCEVRSADDLAAVDGRLRQLLFFHLLNEGIYSSPRGFVVLSLPLT & \\
\hline TAPa & & MRFIGIGSLMNVQITGRPVRSVRDIDAGMNEFKDLLFFHLIEQGIYIARRGYVVLSLPVG & \\
\hline \multirow[t]{2}{*}{ TAEn } & 5 & FQFSGIGSMMTAHATTRPIRTAADIASSNQDAKELFFFDMSAAGIWIARRGFAALNIMIG & 47 \\
\hline & & $:{ }^{\star}{ }^{\star}:{ }^{\star}: \quad: \quad:{ }^{\star} .{ }^{\star}: . \quad:{ }^{\star}:{ }^{\star} .:$ & \\
\hline & & DAGRDAFAEALADFIGEQRALLM------- & \\
\hline & & DAEADEFVAALDAVVTARHALLPVAG---- & \\
\hline & & DAEIDRYVAAIGSFIGGHAALLPRAD---- & \\
\hline TAPa & & DAEMARLEAALASFIERYAHLLPRDDNSDR & \\
\hline \multirow[t]{2}{*}{ TAEn } & 475 & EAEADRFVSAVEEFVTARKALLQP------ & 49 \\
\hline & & $: \star \quad \quad \star: \quad .:=\quad \star \star \star 2$ & \\
\hline
\end{tabular}

TAMe: $\quad \beta$-transaminase from Mesorhizobium sp. LUK; TAPo: glutamate-1-semialdehyde 2,1-aminomutase from Polaromonas sp. JS666; TAVa: aminotransferase class III-fold pyridoxal phosphate-dependent enzyme from Variovorax paradoxus; TAPa: aminotransferase class III-fold pyridoxal phosphate-dependent enzyme from Paraburkholderia gramini.

Figure S2 Graph of the SDS-PAGE of $\omega$-TAEn. 


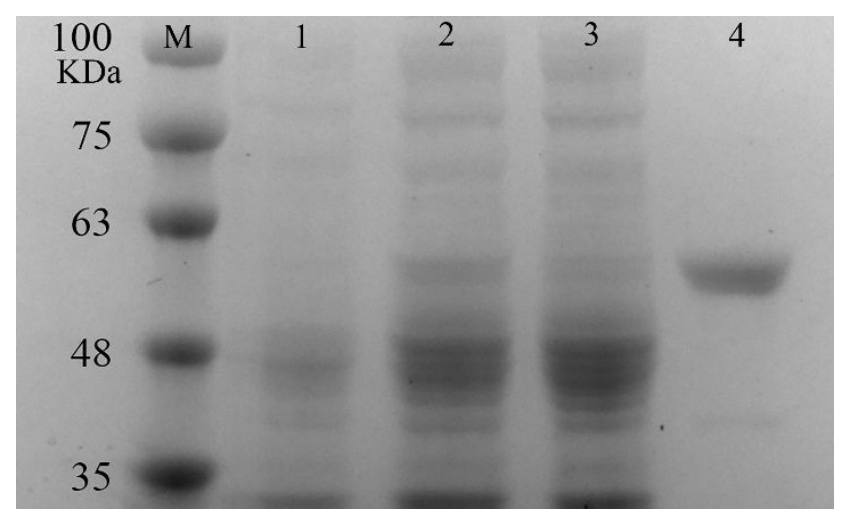

Lanes: (M) protein marker; (1) control; (2) soluble fraction of cell extract; (3) penetrating fluid of purification; (4) purified enzyme.

Figure S3 Graph of temperature effect on tandem reaction between lipase and

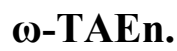

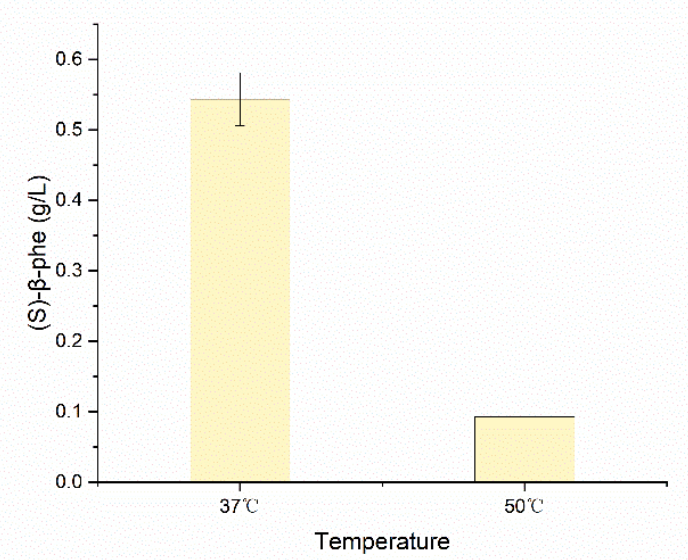

The reaction was carried out in Tris- $\mathrm{HCl}$ buffer $(100 \mathrm{mM}, \mathrm{pH} 8)$ containing ethyl phenylpyruvate $(10 \mathrm{mM})$, PLP $(0.1 \mathrm{mM})$, DMSO $(5 \%, \mathrm{v} / \mathrm{v})$, DL-3-aminobutyric acid $(80 \mathrm{mM})$, lipase $(1 \mathrm{mg} / \mathrm{mL})$ and $\omega-\mathrm{TA}(0.045 \mathrm{mg} / \mathrm{mL})$ at $37^{\circ} \mathrm{C}$ or $50^{\circ} \mathrm{C}$. 
Figure S4 Asymmetric synthesis of (S)- $\beta$-phe with different 3-aminobutyric acid concentrations.

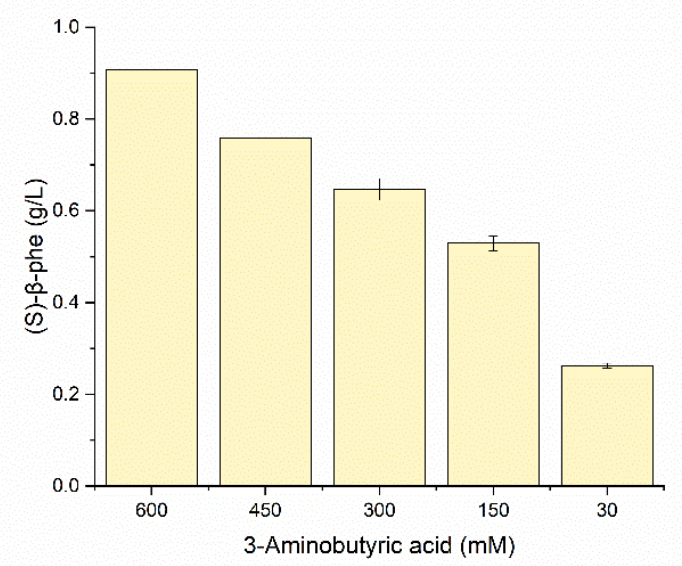

The reaction was carried out in Tris- $\mathrm{HCl}$ buffer $(100 \mathrm{mM}, \mathrm{pH} 8)$ containing ethyl phenylpyruvate $(10 \mathrm{mM}), \operatorname{PLP}(0.1 \mathrm{mM}), \operatorname{DMSO}(5 \%, \mathrm{v} / \mathrm{v}), \mathrm{DL}-3$-aminobutyric acid $(30-600 \mathrm{mM})$, lipase $(1 \mathrm{mg} / \mathrm{mL})$ and $\omega$-TA $(0.045 \mathrm{mg} / \mathrm{mL})$ at $37^{\circ} \mathrm{C}$.

Figure S5 HPLC diagram of the rac- $\beta$-phe sample.

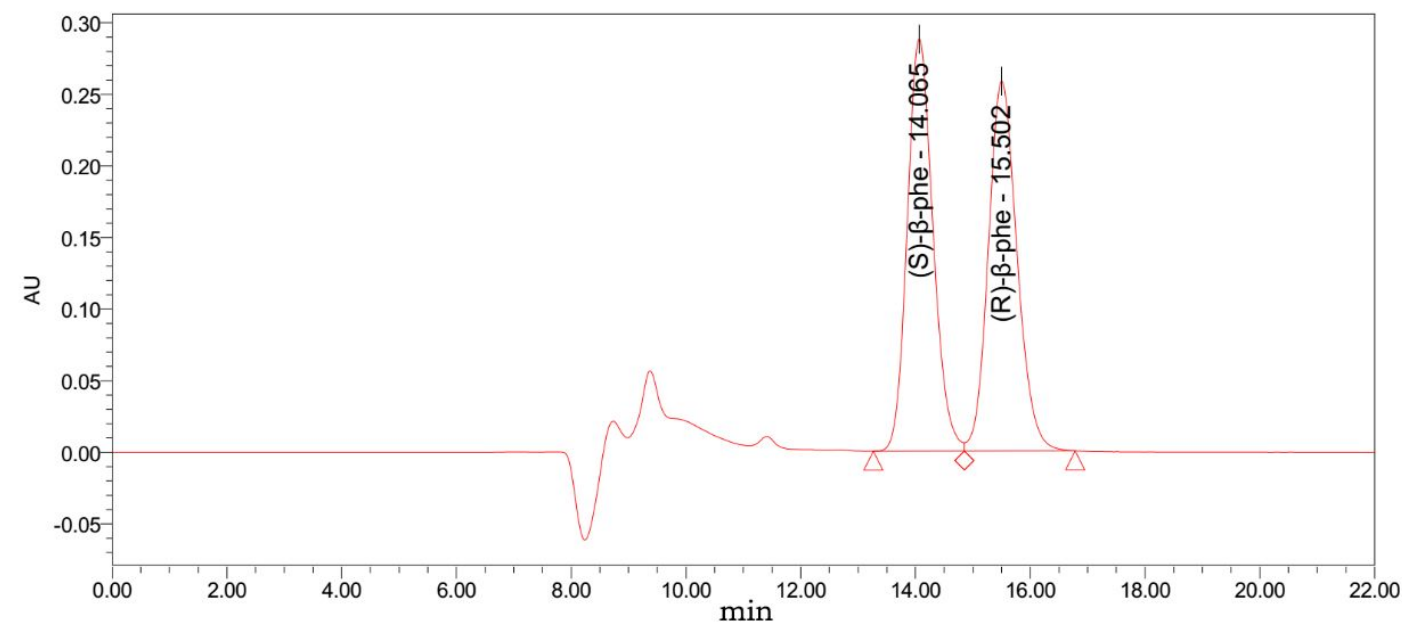


